\title{
Strongly Non-Arrhenius Self-Interstitial Diffusion in Vanadium
}

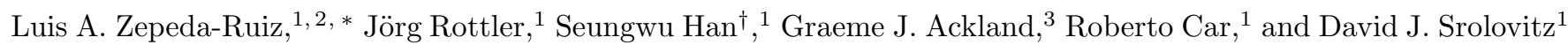 \\ ${ }^{1}$ Princeton Institute for the Science and Technology of Materials (PRISM), Princeton University, Princeton, NJ 08544 \\ ${ }^{2}$ Chemistry and Materials Science Directorate, Lawrence Livermore \\ National Laboratory, P.O. Box 808, L-371, Livermore, CA 94550 \\ ${ }^{3}$ School of Physics, University of Edinburgh, Edinburgh EH9 3JZ, United Kingdom
}

(Dated: August 29, 2018)

\begin{abstract}
We study diffusion of self-interstitial atoms (SIAs) in vanadium via molecular dynamics simulations. The $\langle 111\rangle$-split interstitials are observed to diffuse one-dimensionally at low temperature, but rotate into other $\langle 111\rangle$ directions as the temperature is increased. The SIA diffusion is highly non-Arrhenius. At $T<600 \mathrm{~K}$, this behavior arises from temperature-dependent correlations. At $T>600 \mathrm{~K}$, the Arrhenius expression for thermally activated diffusion breaks down when the migration barriers become small compared to the thermal energy. This leads to Arrhenius diffusion kinetics at low $T$ and diffusivity proportional to temperature at high $T$.

PACS numbers: $66.30 . \mathrm{Fq}, 61.72 . \mathrm{Bb}, 61.82 . \mathrm{Bg}$
\end{abstract}

The creation and migration of self-interstitial atoms (SIAs) are critical for microstructural evolution of materials in a variety of situations, such as in the high energy radiation environment of nuclear reactors [1] and in ion implantation [2]. Although SIA formation energies are much larger than typical thermal energies, they form in abundance during collision cascades induced by impinging energetic particles. SIAs in metals are typically very mobile (i.e., their migration barriers are relatively small) and hence play an important role in controlling the rates of many microstructural processes in such applications, in particular the phenomenon of void swelling.

Since SIA properties and mobilities are very difficult to determine experimentally, one often employs computer simulations [3, 4, 5, 6]. For example, simulations of body centered cubic (bcc) iron (and several other bcc metals), have shown that SIAs preferentially lie along $\langle 110\rangle$ orientations but rotate into $\langle 111\rangle$-directions, where they can migrate easily using the crowdion configuration as transition state. Other simulations have suggested that SIA migration in vanadium is very similar to that in $\mathrm{Fe}$ 7, $8,9,10$. However, these empirical interatomic potential-based simulations are not consistent with recent first principles calculations that clearly show that the lowest energy SIA configuration in $\mathrm{V}$ is a $\langle 111\rangle$ split interstitial, rather than the $\langle 110\rangle$-split configuration found in $\mathrm{Fe}$ 11]. Interestingly, the first principles calculations also revealed that the $\langle 111\rangle$-oriented SIA migration energy is extraordinarily small $(\leq 0.01 \mathrm{eV})$, which explains the experimental observation of diffusion down to $4 \mathrm{~K}$ [12]. SIA migration in Fe and $\mathrm{V}$ must therefore differ in their microscopic mechanisms.

We perform a series of molecular dynamics (MD) sim-

${ }^{\dagger}$ current address: Department of Physics, Ewha Womans University, Seoul 120-750, Korea. ulation of SIA migration in $\mathrm{V}$ using an improved interatomic potential for V 13] (refit to experimental and first principles data 11 to reproduce the stable interstitial configuration) to address this discrepancy. In particular, we examine SIA diffusion as a function of temperature to determine the SIA migration mechanisms. We find that while SIA migration in V is similar to that in bcc Fe in many respects, its temperature dependence is highly unusual, exhibiting strongly non-Arrhenius behavior and correlation effects. It is this non-Arrhenius behavior that is the focus of the present Letter.

The SIA is introduced in the form of a stable $\langle 111\rangle$ split interstitial and equilibrated for 10 ps at fixed temperature using a Langevin thermostat. The simulation was then switched to a microcanonical (NVE) ensemble in order to study SIA migration dynamics. Simulations were run at temperatures between 100 and $2000 \mathrm{~K}$ in a cubic simulation cell of edge length $10 a_{0}$, where $a_{0}$ is the temperature dependent-lattice parameter (the linear thermal expansion coefficient was $\left.8.4 \times 10^{-6} \mathrm{~K}^{-1}\right)$. The diffusivity was measured by averaging over several $1 \mathrm{~ns}$ simulations. The interstitial position was identified by dividing the space into Wigner-Seitz (W-S) cells centered around each perfect crystal lattice site. Interstitials are located in W-S cells containing more than one atom.

Representative trajectories of the $\langle 111\rangle$-split interstitial center of mass, collected over the whole $1 \mathrm{~ns}$ simulations, are shown in Fig. 1 For each temperature, more than 1000 jumps were observed, where an SIA jump is the exchange of an atom between neighboring W-S cells. As seen in Fig. 1 the interstitial migration mechanism is strongly temperature dependent. For low and intermediate temperatures $(100-600 \mathrm{~K})$ the $\langle 111\rangle$-split interstitial executes a fully one-dimensional (1D) random walk along a $\langle 111\rangle$-direction during the 1 ns simulation, as shown in Fig. 1(a). At $T \sim 700 \mathrm{~K}$, the $\langle 111\rangle$-split interstitial begins to make infrequent rotations from one $\langle 111\rangle$ - to another $\langle 111\rangle$-direction. This results in a three-dimensional 

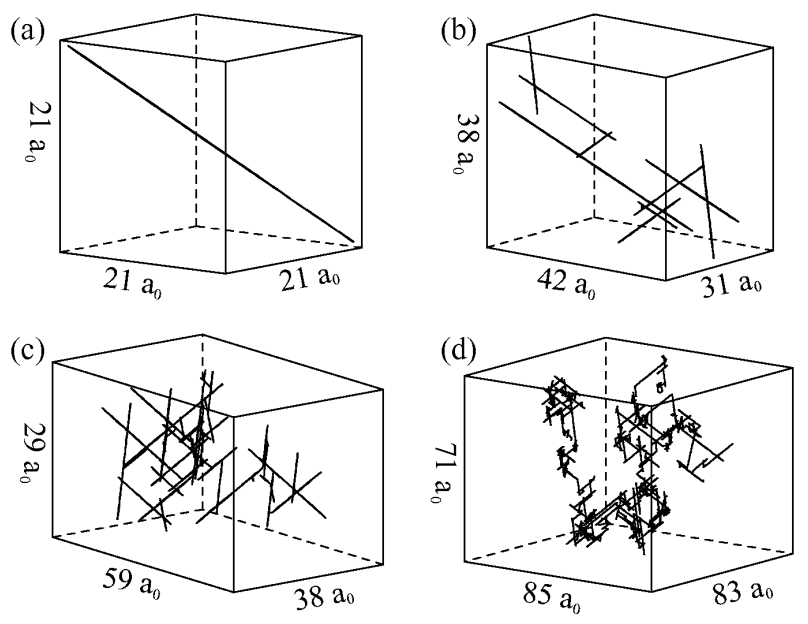

FIG. 1: Typical trajectories of migrating SIAs for temperatures of (a) $300 \mathrm{~K}$, (b) $700 \mathrm{~K}$, (c) $900 \mathrm{~K}$ and (d) $1400 \mathrm{~K}$.

(3D) trajectory that consists of long 1D random walk segments with abrupt reorientations, as seen in Fig. 1(b). As the temperature increases, the frequency of the rotation events increases and the lengths of the $1 \mathrm{D}$ trajectory segments decrease. At high temperatures, the rotation events become very frequent, leading to nearly isotropic diffusion (Fig. 1(d)).

Although these trajectories appear to be qualitatitively similar to those reported for other bcc metals (i.e. Fe and Mo) 4, 14, 15, 16] (cf. Fig. 1(d) and Fig. 5 in 15]), they differ in the elementary migration mechanism. The stable interstitial is the $\langle 111\rangle$-split configuration in $\mathrm{V}$, but the $\langle 110\rangle$-split configuration in Fe and Mo. In the $\mathrm{Fe}$ and Mo cases, the split interstitial sits in the $\langle 110\rangle$ orientation until it is thermally activated into one of the $\langle 111\rangle$-directions where it can migrate easily before returning to a $\langle 110\rangle$-orientation [15]. There are no relaxation events of this type in interstitial migration in $\mathrm{V}$. Here, the stable $\langle 111\rangle$-split interstitial migrates long distances and only requires significant thermal activation to reorient or rotate.

The temperature dependence of the rate of rotation of the split interstitials from one $\langle 111\rangle$ direction to another, $\omega_{r}$, in $\mathrm{V}$ is shown in Fig. 2. The data is well described by a conventional Arrhenius fit of the form $\omega_{r}=\nu_{0} \exp \left[-\Delta E_{r} / k_{B} T\right]$, suggesting that rotation is a simple thermally activated process. The activation energy, $\Delta E_{r}=0.44 \mathrm{eV}$, is consistent with the energy difference between the $\langle 111\rangle$ and $\langle 110\rangle$ configurations computed in first-principles $(0.35 \mathrm{eV})$ and static calculations using the new interatomic potential $(0.4 \mathrm{eV})$ 13].

The diffusivity $D$ (solid symbols) of the $\langle 111\rangle$-split interstitial is shown in Fig. B for a temperature range between $100 \mathrm{~K}$ and $2000 \mathrm{~K}$. $D$ was determined from $D=\left\langle R^{2}(t)\right\rangle / 2 t$ for $1 \mathrm{D}$ diffusion, where the mean squared displacement $\left\langle R^{2}(t)\right\rangle$ was calculated from the trajectory

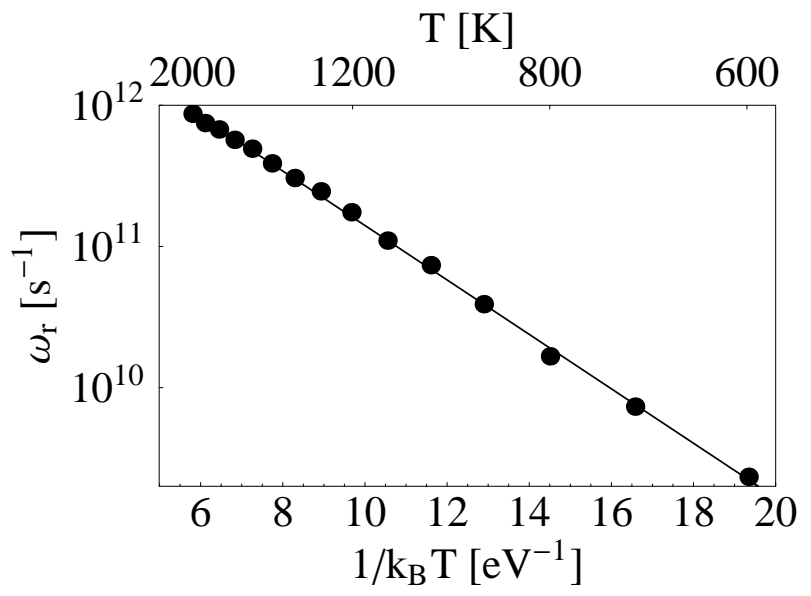

FIG. 2: Frequency of rotations as a function of temperature. The solid line is an Arrhenius fit to the data with slope $\Delta E_{r}=$ $0.44 \mathrm{eV}$, preexponential factor $\nu_{0}=1.3 \times 10^{13} \mathrm{~s}^{-1}$.

using standard averaging procedures 17. Note that although the rotations at higher $T$ change the dimensionality of the diffusion path, they do not contribute to transport and hence the diffusion mechanism is always one-dimensional. If the diffusivity were Arrhenius $\left(D / a_{0}^{2}=\nu_{0} \exp \left[-\Delta E_{d} / k_{B} T\right]\right)$, the data in Fig. 3 would lie along a straight line. This is clearly not the case; Fig. 3 shows pronounced curvature - especially at high temperature. Although Arrhenius behavior is widely expected for diffusion in the solid state, it is clearly inapplicable here.

Non-Arrhenius behavior can have several different origins. The energy barrier could simply change with temperature as a result of thermal expansion, as has been argued for the self-diffusion in bcc metals via a vacancy mechanism [18, 19]. The magnitude of the observed deviations from Arrhenius behavior is too large to attribute to such a mechanism. The existence of multiple reaction pathways with different energy barriers can also lead to curvature in Fig. 3 However, detailed examination of the atomic configuration during diffusion shows that there is no change in diffusion mechanism over the entire temperature range. Although rotations are first observed at $\sim 700 \mathrm{~K}$ within the $1 \mathrm{~ns}$ duration of the simulations, strong deviations from Arrhenius behavior are observed already at lower temperatures. A third possibility is that the degree of correlations in the diffusion process (i.e., particle jumps retain memory and the random walk is non-Markovian) is temperature dependent. Indeed, examination of the SIA trajectories show that the $\langle 111\rangle$ interstitial has a higher probability of jumping back in the direction from whence it came, rather than forward along the same direction. At high temperatures, by contrast, this effect appears to be reversed.

We quantified this observation by measuring a correlation factor for split interstitial diffusion $f$, defined as 


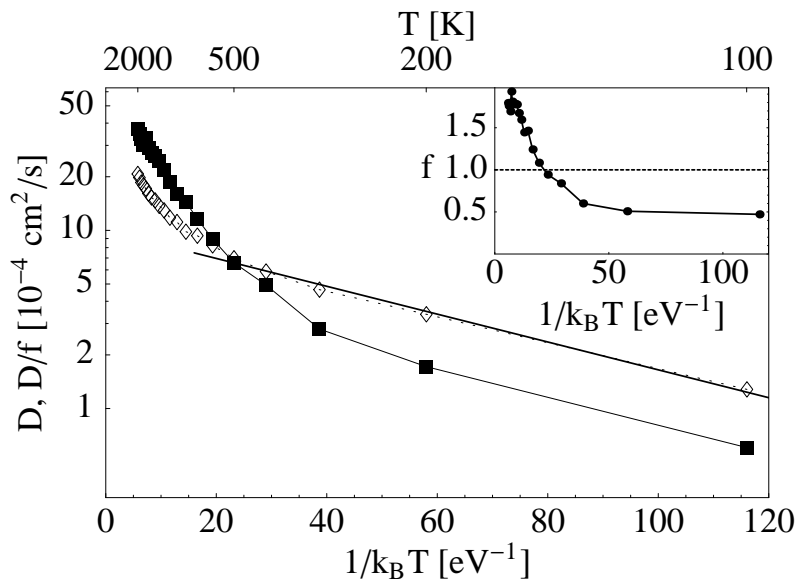

FIG. 3: A plot of the diffusivity of the $\langle 111\rangle$-split interstitial in the form suggested by the Arrhenius relation. The filled symbols ( $)$ correspond to the measured diffusivity $D$ and open symbols $(\diamond)$ to $D$ normalized by the correlation factor $f$ (see text). The straight line is a low temperature fit to the $D / f$ data, corresponding to $\Delta E_{d}=0.018 \mathrm{eV}$ and a preexponential factor of $\nu_{0}=1.5 \times 10^{12} \mathrm{~s}^{-1}$ in the Arrhenius form. Statistical errors are of order the symbol size. The inset to the figure shows the variation of $f$ as a function of $1 / k_{B} T$.

$f=2 D / D_{b}$, where $D_{b}$ is the "bare" diffusion constant defined as $D_{b}=l_{0}^{2} n$. Here, $n$ is the mean number of jumps/second and $l_{0}=\sqrt{3} a_{0} / 2$ is the jump length (nearest neighbor distance in the bcc lattice). If the interstitial trajectory is described by a sequence of jump vectors $\vec{l}_{i}$, the correlation factor $f$ is alternatively given by $f=1+2 \sum_{i}^{n-1}\left\langle\vec{l}_{i} \cdot \vec{l}_{0}\right\rangle / l_{0}^{2}$, i.e. $f=1$ for an uncorrelated random walk. The inset to Fig. [3 shows the variation of $f$ with $1 / k_{B} T$. At low $T(T \leq 600 \mathrm{~K})$, SIA motion is anticorrelated $(f<1)$. The effect of the correlations on the diffusivity can be isolated by plotting $D / f$ rather than $D$ in Fig. 3 (open symbols). The low temperature $D / f$ data $(T \leq 500 K)$ lie along a nearly straight line with slope $\Delta E_{d}=0.018 \mathrm{eV}$. Hence, the temperature dependent correlation factor explains the relatively weak deviations from Arrhenius behavior at low $T$. The unusually small migration energy is consistent with experimental observations [12] and the first principle estimate [11. One possible origin of the anticorrelations is that the finite relaxation time of the local environment around the SIA becomes less important as the thermal energy of the system increases.

As the temperature is increased beyond $300 \mathrm{~K}$, the correlation factor rises quickly to a value greater than unity. $f>1$ is very unusual and may be thought of as correlated interstitial hopping over several barriers without completely thermalizing in between. This interpretation is consistent with the fact that the correlation corrected diffusivity, $D / f$, only yields a straight line at low $T$ but not at high $T$. We note that where $D / f$ is rising quickly, the energy barrier $\Delta E_{d}$ obtained in the low

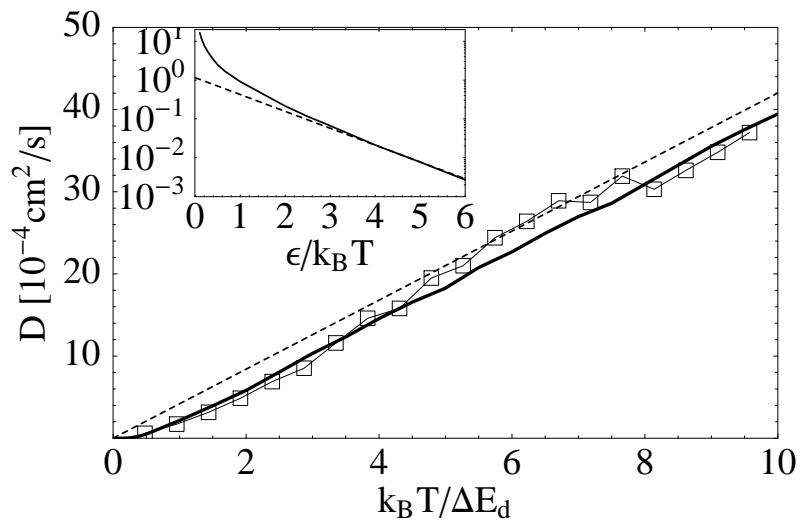

FIG. 4: The diffusivity $D(\boldsymbol{\square})$ from Fig. 3 as a linear function of $T$, normalized by $\Delta E_{d}$. The thick solid line represents the diffusivity of a particle in a sinusoidal potential at finite temperature as described by Eq. (1) for a value of $\gamma=0.1 \tau^{-1}$ where $\tau=\sqrt{m \sigma^{2} / \epsilon}$. The dashed line is the free particle limit of the same model, $D=k_{B} T / \epsilon m \gamma$. The inset shows an Arrhenius plot of the diffusivity of the particle in the sinusoidal potential as a function of $\epsilon / k_{B} T$ as a solid line and the straight dashed line has slope one.

temperature regime (where the behavior is Arrhenius) is smaller than the thermal energy. This is the source of the multiple interstitial hops at high $T$. Conventional derivations of activated escape over barriers [20] usually assume $\Delta E_{d} \gg k_{B} T$.

Rather than trying to apply the Arrhenius description to interstitial self-diffusion in this system, a more general model is the motion of a particle in a periodic potential at all temperatures (from $k_{B} T \ll \Delta E_{D}$ to $k_{B} T \gg \Delta E_{D}$ ). In the limit that the barrier height is completely negligible relative to the energy of the heat bath (i.e., a free particle), standard arguments predict $D=k_{B} T / m \gamma$, where $m$ is the mass of the particle and $\gamma$ a relaxation time scale associated with a velocity-dependent friction force. Since this free particle diffusion coefficient is expected to be a linear function of $T$, we replot the data from Fig. B] on a linear temperature scale (Fig. (4i). In this representation, the data is nearly linear, albeit with weak curvature at low temperature. This suggests that SIA diffusion in $\mathrm{V}$ is free particle like at high temperature $(D \sim T)$, but follows the normal Arrhenius, hopping dynamics at low $T\left(D \sim e^{-\Delta E_{D} / k_{B} T}\right)$. The deviation from linearity at low temperature and the deviation from Arrhenius behavior at high temperature suggests that a cross-over is occuring between the free and hopping particle limits.

In order to better understand this transition, we explicitly consider a simple one-dimensional particle of mass $m$ diffusing in a sinusoidal potential by numerically solving the Langevin equation

$$
m \ddot{x}-\gamma \dot{x}=\epsilon / 2 \cos [x / \sigma]+\eta,
$$

where $\eta$ is a Brownian white noise. Inserting values of $m$ and $\sigma$ for $\mathrm{V}$ and $\epsilon=\Delta E_{d}$, this model yields nearly 
linear diffusivity for $1<k_{B} T / \epsilon<10$ for reasonable values of $\gamma$, followed by a crossover into Arrhenius behavior (see inset of Fig. 4) for $k_{B} T / \epsilon \lesssim 1$. Changing the value of $\gamma$ shifts the temperature at which the transition from the particle hopping to free particle behavior is observed. This excellent agreement between the MD results and model predictions demonstrates that the observed strongly non-Arrhenius interstitial diffusion in $\mathrm{V}$ is a direct consequence of the relative magnitudes of the activation energy and the thermal energy.

It is interesting to compare the situation described here for $\mathrm{V}$ to that for bcc Fe. The crowdion mechanism enabling the easy interstitial migration along $\langle 111\rangle$ directions in $\mathrm{V}$ is also available in $\mathrm{Fe}$, and estimates for the migration barrier $\Delta E_{d}=0.04 \mathrm{eV}$ 4 are similar to the $\mathrm{V}$ case. However, measurements of the apparent activation energy for diffusivity, analogous to the one presented here (albeit over a smaller temperature range between $700 \mathrm{~K}$ and $1200 \mathrm{~K}$ ) yield much larger values of $\Delta E_{d}=0.12$ $\mathrm{eV}$ [16] or $\Delta E_{d}=0.17 \mathrm{eV}$ [21]. This larger effective barrier is due to the fact that the $\langle 111\rangle$-split interstitial must be thermally excited from the $\langle 110\rangle$-state, i.e. the easy-diffusion configuration is not populated at all times as in V. The fraction of time during which the $\langle 111\rangle$ split interstitial is available for transport is given by $F=$ $P_{\langle 111\rangle} /\left(P_{\langle 111\rangle}+P_{\langle 110\rangle}\right)$, where $P_{\langle 111\rangle} \sim \exp \left[-\Delta E_{f} / k_{B} T\right]$ and $P_{\langle 110\rangle} \sim \exp \left[-\Delta E_{b} / k_{B} T\right]$ are probabilities for the interstitial to transform from the $\langle 110\rangle$ state to the $\langle 111\rangle$ state and back, respectively. Therefore, the interstitial diffusivity in $\mathrm{Fe}$ is the product of the diffusivity that the interstitial would have if it was always in the $\langle 111\rangle$ orientation (like in $\mathrm{V}$ ) and $F$. For $\Delta E_{f} \gg \Delta E_{b}$, the effective activation energy for interstitial diffusivity in $\mathrm{Fe}$ is $\sim\left(\Delta E_{D}+\Delta E_{f}\right)$, but for $\Delta E_{b} \gg \Delta E_{f}$, it is $\sim \Delta E_{D}$. Clearly, this implies for Fe that $\Delta E_{f} \gg \Delta E_{b}$.

Molecular dynamics simulations of self-interstitial diffusion in bcc $\mathrm{V}$ were performed over an unusually wide temperature range $(100-2000 \mathrm{~K})$. Interstitial atoms in the $\langle 111\rangle$-split configuration migrate very fast onedimensionally along $\langle 111\rangle$ directions during the $1 \mathrm{~ns}$ simulations. As $T$ is increased above $600 \mathrm{~K}$, rotations of the split-interstitial from one $\langle 111\rangle$ orientation to another occur with increasing regularity. The rotations can be described by Arrhenius kinetics with activation energy $\Delta E_{r}=0.44 \mathrm{eV}$. At temperatures $T<600 \mathrm{~K}$, the diffusion exhibits significant anticorrelations. An Arrhenius analysis of the data (corrected for these anticorrelations) yields a very small migration energy barrier $\Delta E_{d}=0.018$ $\mathrm{eV}$. For $T>600 K, \Delta E_{d}$ is much smaller than the thermal energy and the Arrhenius expression is no longer applicable. The diffusivity then crosses over from Arrhenius to free particle type diffusion with increasing $T$. The fact that this type of diffusion is a linear function of $T$ rather than Arrhenius, as usually assumed, could have important implications for predicting the lifetimes of reactor components in vanadium and other bcc metals.

We thank A. F. Voter, G. H. Gilmer, and J. A. Caro for useful discussions. This work was performed under the auspices of the U. S. Department of Energy, Office of Fusion Enery Sciences (DE-FG02-01ER54628) and Lawrence Livermore National Laboratory under Contract No. W-7405-Eng-48.

* corresponding author: zepedaruiz1@llnl.gov

[1] F. W. Young, J. R. Cost, A. Nowick, and J. O. Stiegler, Mater. Sci. Eng. 35, 91 (1978).

[2] K. Nordlund, J. Keinonen, M. Ghaly, and R. S. Averback, Nature 398, 49 (1999).

[3] T. Díaz de la Rubia and M. W. Guinan, Phys. Rev. Lett. 66, 2766 (1991).

[4] B. D. Wirth, G. R. Odette, D. Maroudas, and G. E. Lucas, J. Nucl. Mater. 244, 185 (1997).

[5] N. Soneda and T. Díaz de la Rubia, Phil. Mag. A 78, 995 (1998).

[6] B. D. Wirth, G. R. Odette, D. Maroudas, and G. E. Lucas, J. Nucl. Mater. 276, 33 (1999).

[7] A. M. Minashin and V. A. Ryabov, J. Nucl. Mater. 233237, 996 (1996).

[8] K. Morishita, N. Sekimura, and T. Díaz de la Rubia, J. Nucl. Mater. 248, 400 (1997).

[9] K. Morishita, T. Díaz de la Rubia, E. Alonso, N. Sekimura, and N. Yoshida, J. Nucl. Mater. 283, 753 (2000).

[10] K. Morishita, T. Díaz de la Rubia, and A. Kimura, Nucl. Instr. Meth. Phys. Res. B 180, 66 (2001).

[11] S. Han, L. A. Zepeda-Ruiz, G. J. Ackland, R. Car, and D. J. Srolovitz, Phys. Rev. B 66, 220101 (2002).

[12] R. R. Coltman Jr., C. E. Klabunde, J. K. Redman, and J. M. Williams, Radiat. Eff. 24, 69 (1975).

[13] S. Han, L. A. Zepeda-Ruiz, G. J. Ackland, R. Car, and D. J. Srolovitz, J. Appl. Phys. 93, 3328 (2002).

[14] Yu. N. Osetsky, M. Victoria, A. Serra, S. I. Golubov, and V. Priego, J. Nucl. Mater. 251, 34 (1997).

[15] R. C. Pasianot, A. M. Monti, G. Simonelli, and E. J. Savino, J. Nucl. Mater. 276, 230 (2000).

[16] J. Marian, B. D. Wirth, J. M. Perlado, G. R. Odette, and T. Díaz de la Rubia, Phys. Rev. B 64, 094303 (2001).

[17] M. W. Guinan, R. N. Stuart, and R. J. Borg, Phys. Rev. B 15, 699 (1977).

[18] K. Eftaxias and V. Hadjicontis, Phys. Stat. Sol. B 156, 393 (1989).

[19] G. Neumann and D. L. Beke, Phys. Stat. Sol. B 161, K5 (1990).

[20] P. Haenggi, P. Talkner, and M. Borkovec, Rev. Mod. Phys. 62, 251 (1990).

[21] N. Soneda and T. Díaz de la Rubia, Phil. Mag. A 81, 331 (2001). 